\title{
On the density of EKOs and related objects
}

\author{
W. J. Altenhoff ${ }^{1}$, F. Bertoldi ${ }^{1,2}$, K. M. Menten ${ }^{1}$, and C. Thum ${ }^{3}$ \\ 1 Max-Planck-Institut für Radioastronomie, Auf dem Hügel 69, 53121 Bonn, Germany \\ e-mail: author@mpifr-bonn.mpg.de \\ 2 Radioastronomisches Institut der Universität Bonn, Auf dem Hügel 71, 53121 Bonn, Germany \\ e-mail: bertoldi@astro.uni-bonn.de \\ 3 IRAM, Domaine Universitaire de Grenoble, 300 rue de la piscine, 38406 St. Martin d'Hères, France \\ e-mail: thum@iram.fr
}

Received 25 May 2005 / Accepted 11 August 2005

\begin{abstract}
Recently published mass determinations of EKO binaries, combined with photometric size determinations, allow to derive a mean density of the distant minor planets of $\approx 0.2 \mathrm{~g} \mathrm{~cm}^{-3}$. This agrees well with the nuclear density of $1 \mathrm{P} / \mathrm{Halley}$ of $0.26 \mathrm{~g} \mathrm{~cm}^{-3}$, determined in the Giotto mission, and it suggests that these low density objects are essentially undifferentiated planetesimals.
\end{abstract}

Key words. minor planets: general - comets: individual: Halley

\section{Introduction}

At the end of the last century a new group of faint Distant Minor Planets (DMPs) was detected, including the EdgeworthKuiper-Objects (EKOs), the Scattered Disk Objects (SDOs), and the Centaurs; altogether 1018 such objects are catalogued today (Parker 2005). Most likely the short period comets, such as $\mathrm{P} / \mathrm{Halley}$, originate from the DMPs. The DMPs are probably remnant planetesimals of the early solar nebula (Weissmann 1995). With optical telescopes many of the properties derived from the reflected sun light (position, absolute magnitude, reflection spectrum) can be measured precisely, but the size and albedo must be derived from the thermal emission by assuming equilibrium with insolation. The thermal emission is so faint that until today it could be detected from only 8 DMPs (Altenhoff et al. 2004). Knowledge of the object size and mass is crucial for a general interpretation, and if their direct measurement is impossible, they are often estimated by assuming specific values for the geometric red albedo, $p=0.04$, and for the average density, $\rho=1.0 \mathrm{~g} \mathrm{~cm}^{-3}$. This value for the albedo was originally based on observations of short period-comets and Centaurs (Jewitt \& Luu 2000), and as the default density that of water ice was assumed. With this the observed size and number distributions yield an estimate for the total mass of the Kuiper belt (Jewitt et al. 1996) and scattered disk (Trujillo et al. 2000) objects of $\approx 0.25$ Earth masses.

The first 8 DMP albedo measurements showed significant source-to-source variations, with an average value twice that of the assumed canonical value (Altenhoff et al. 2004). In light of these measurements we here reexamine the sparse KBO data to reassess the assumption on the objects' densities and the resulting total mass estimate.

\section{Comparison of published albedo values}

Recent observations of EKO binary orbits by Veillet et al. (2002), Osip et al. (2003), Noll et al. (2004a, 2004b), and Margot et al. (2004) allowed mass determinations of the respective systems. With an adopted lower density limit $\rho=$ $1.0 \mathrm{~g} \mathrm{~cm}^{-3}$ they calculated the size, and in combination with the absolute magnitude, $H$, the geometric albedo values, $p$, listed in Table 1. The uncertainties of the albedos are about $10 \%$, although only two of the papers quantify them.

For a comparison with the binaries, Table 1 also lists the thermally determined albedos of six other EKOs, four Centaurs, and one short period comet. The Centaurs have sizes comparable to EKOs, but are not affected by an albedo selection bias: since their optical brightness is a selection criterion for size measurements, distant EKOs with high albedos are preferentially selected over those with low albedos.

Most authors reporting mass determinations of binary EKOs limit the density estimates to a "plausible" range between $2 \mathrm{~g} \mathrm{~cm}^{-3}$ (Pluto) and $1 \mathrm{~g} \mathrm{~cm}^{-3}\left(\mathrm{H}_{2} \mathrm{O}\right)$ and, recognizing that the binary albedos were systematically higher, suggested that EKO binaries were in some way special. For example, Margot et al. (2004) suggested that such high albedos might come from a continuous "collisional resurfacing".

From the data in Table 1 the average object density can be estimated in two different ways, both of which are compared in Table 2 with the density of P/Halley (derived from the size information obtained in the Giotto mission (Rickman 1990)). For Centaurs and EKOs the sizes and geometric albedos derived by thermal photometry (Altenhoff et al. 2004) are free from systematic errors. For binary EKOs the masses derived from their orbits do also not have systematic errors. Thus a 
Table 1. Published albedo values.

\begin{tabular}{lllll}
\hline \hline Name & $H^{*}$ & $p$ & rms $^{* *}$ & Ref. \\
\hline EKO binaries & & & & \\
1998 WW31 & 6.1 & 0.057 & 0.005 & (V1) \\
2001 QT297 & 5.5 & 0.09 & - & (O1) \\
1997 CQ29 & 6.6 & 0.37 & - & (N1) \\
1999 RZ253 & 5.9 & 0.12 & 0.01 & (N2) \\
1999 TC36 & 4.7 & 0.23 & - & (M1) \\
1998 SM165 & 5.7 & 0.15 & - & (M1) \\
2001 QC298 & 6.0 & 0.076 & - & (M1) \\
1997 CQ29 & 6.4 & 0.41 & - & (M1) \\
Average & & 0.188 & & \\
\hline Centaurs & & & & \\
Chiron & 6.5 & 0.13 & 0.004 & (A1) \\
Chariklo & 6.4 & 0.055 & 0.008 & (A2) \\
Chariklo & 6.4 & 0.045 & 0.010 & (J1) \\
Asbolus & 8.4 & 0.12 & 0.03 & (F1) \\
Pholus & 7.0 & 0.044 & 0.013 & (D1) \\
Average & & 0.079 & & \\
\hline EKOs & & & & \\
1999 TC36 & 4.9 & 0.05 & 0.01 & (A3) \\
Varuna & 3.7 & 0.06 & 0.02 & (A3) \\
Varuna & 3.7 & 0.07 & 0.03 & (A3) \\
Ixion & 3.2 & $\geq 0.15$ & - & (A3) \\
2002 AW197 & 3.3 & 0.09 & 0.02 & (A3) \\
Quaoar & 2.6 & 0.10 & 0.03 & (A3) \\
Sedna & 1.5 & $\geq 0.18$ & - & (B1) \\
Average** & & 0.075 & & \\
\hline Short period comets & & & \\
P/Halley & & 0.04 & 0.01 & (W1) \\
\hline & & & &
\end{tabular}

Notes:

* Absolute magnitude, $H$, and geometric albedo, $p$, from listed reference or, if no reference is given, taken from CBAT (2004).

${ }^{* *}$ Missing error limits are estimated to about $15 \%$.

*** Albedo average at $250 \mathrm{GHz}$ of detected EKOs. Bright objects with $H \leq 2.5$ (e.g. Sedna, $2004 \mathrm{DW}$, etc.) are not included in the density determination, because they likely belong to a different physical class of objects.

References: (V1) Veillet et al. (2002); (O1) Osip et al. (2003); (N1) Noll et al. (2004a); (N2) Noll et al. (2004b); (M1) Margot et al. (2004); (A1) Altenhoff \& Stumpff (1995); (A2) Altenhoff et al. (2001); (J1) Jewitt \& Kalas (1998); (F1) Fernandez et al. (2002); (D1) Davies et al. (1993); (A3) Altenhoff et al. (2004); (B1) Brown et al. (2004); (W1) Whipple (1986).

Table 2. Density estimates for DMP.

\begin{tabular}{lcrl}
\hline \hline Method & $\begin{array}{c}\text { Density } \\
{\left[\mathrm{g} \mathrm{cm}^{-3}\right]}\end{array}$ & $\begin{array}{c}\text { rms } \\
\end{array}$ & Comment \\
\hline (a) Direct & 0.14 & 0.04 & 1999 TC36 \\
(b) Statistical & 0.25 & 0.15 & bin./single ratio \\
(c) P/Halley & 0.26 & 0.15 & non-grav. forces \\
\hline
\end{tabular}

combination of thermal emission and orbit period measurements allows to derive the actual density of the EKOs. With the direct mass determinations of the binary EKOs their geometric albedos are given in the literature assuming a density of $\rho=1$; the albedo scales with the density as $p \propto \rho^{2 / 3}$. The intercept of the albedo function with the thermally determined albedo marks the EKO density.

(a) Direct method: the binary 1999 TC36 is so far the only EKO with a thermal size determination, allowing a direct density determination with the scheme described above. So the resulting density of this binary is $\rho=(0.12 \pm$ $0.04) \mathrm{g} \mathrm{cm}^{-3}$. This is the first density determination of an EKO, derived without any further assumption. We used the albedo accuracy from the thermal observations to estimate the uncertainty of the density.

(b) Statistical approach: the method used for the single binary EKO can also be applied to the mean albedo value of a group of binaries, e.g. for that in Table 1. Since we do not have for this group the mean thermally determined albedo we substitute the mean albedo of the single EKOs, assuming that the average albedo is the same for all EKOs. Table 1 shows that the average albedo of the binary EKOs, derived with an assumed density of $1 \mathrm{~g} \mathrm{~cm}^{-3}$, is about twice that of the average albedo of single EKOs and Centaurs. From this we then find an ensemble average density of $(0.25 \pm 0.15) \mathrm{g} \mathrm{cm}^{-3}$. The uncertainty of this density is derived from the half power width of the albedo distribution.

(c) Halley: short period comets possibly originate in the Kuiper belt (e.g. Duncan \& Levison 1997) and have a relatively short life time compared to DMPs. They move in dynamically unstable orbits and experience high mass loss. At each apparition Comet 1P/Halley, e.g., loses $3.5 \times 10^{11} \mathrm{~kg}$ of volatile ices (Whipple 1986), which corresponds to a $\sim 10 \mathrm{~m}$ mantle depth. This high mass loss may explain the difference in color and albedo (Jewitt 2002) between EKOs and short period comets. The mass loss does, however, not affect the internal constitution of an object's nucleus. Thus, the low density of Halley's nucleus, $0.26 \pm 0.15 \mathrm{~g} \mathrm{~cm}^{-3}$, that was derived from the molecular production rates and the resulting orbit changes (Rickman 1990), should be similar to the average density of the DMPs. The uncertainties to the density given by Rickman are lower and higher estimates, not statistical error limits.

The quality of the three results in Table 2, indicated by the error limits, is quite different, although within the error limits they do agree. Given how e.g. the upper and lower "bounds" of Comet 1P/Halley (Rickman 1990) were obtained, it is not appropriate to calculate a weighted average, which presumes statistical errors. We therefore estimate that the most likeley density is given by a value consistent with all three estimates, and the uncertainty of this estimate is given by the total variance of the three measurements: $\rho \approx 0.2_{-0.1}^{+0.2} \mathrm{~g} \mathrm{~cm}^{-3}$.

\section{Interpretation and conclusion}

Combining the mass determination of a binary EKO with thermal measurements of sizes and albedos of EKOs and a comet we find a low average density that may be typical for all DMPs. With this density the binary EKOs no longer seem as a special class of object. 
The total mass estimates for the Kuiper and scattered disk objects were previously done under the assumptions that $r=$ 0.04 and $\rho=1.0 \mathrm{~g} \mathrm{~cm}^{-3}$. Using our new average values, $r=$ 0.08 and $\rho=0.2 \mathrm{~g} \mathrm{~cm}^{-3}$, we estimate the total mass of the minor planet populations to $1.0 \times 10^{26} \mathrm{~g}$ or $1.7 \%$ of the mass of the Earth, which is 7 percent of the previous estimates.

The derived density of DMPs and of the one short period comet for which it has been measured is so low that it is unlikely that they contain nuclei with densities of $\geq 1.0 \mathrm{~g} \mathrm{~cm}^{-3}$, as would be expected after chemical and thermal processing. This strengthens the interpretation that all these objects are still pristine, undifferentiated planetesimals.

The typical albedos and densities of the DMPs and the Pluto Charon-Binary are so different that it seems unlikely that the latter is a member of the DMP population.

Although tantalizing, these results are in strong need of confirmation through accurate thermal measurements of a larger number of binary EKOs.

\section{References}

Altenhoff, W. J., \& Stumpff, P. 1995, A\&A, 293, L41

Altenhoff, W. J., Menten, K. M., \& Bertoldi, F. 2001, A\&A, 366, L9

Altenhoff, W. J., Bertoldi, F., \& Menten, K. M. 2004, A\&A, 415, 771

Brown, M. E., Trujillo, C. A., Rabinowitz, D., et al. 2004, BAAS, 36, 1068
CBAT, List of Transneptunian Objects 2004, Central Bureau for Astronomical Telegrams, Cambridge, USA

Davies, J., Spencer, J., Sykes, M., et al. 1993, IAU Circ., 5698

Duncan, M. J., \& Levison, H. F. 1997, Science, 276, 12

Fernandez, Y. R., Jewitt, D. C., \& Sheppard, S. S. 2002, AJ, 123, 1050 Jewitt, D. C. 2002, AJ, 123, 1039

Jewitt, D. C., \& Kalas, P. 1998, ApJ, 115, L103

Jewitt, D. C., \& Luu, J. 2000, Physical Nature of the Kuiper Belt, in Protostars and Planets IV, ed. V. Mannings, \& A. P. Boss (Univ. Arizona Press), 1201

Jewitt, D. C., Luu, J., \& Chen, J. 1996, AJ, 112, 1225

Margot, J. L., Brown, M. E., Trujillo, C. A., \& Sari, R. 2004, BAAS, 36, 1081

Noll, K. S., Stephens, D. C., Grundy, W. M., et al. 2004a, AJ, 128, 2547

Noll, K. S., Stephens, D. C., Grundy, W. M., \& Griffin, I. 2004b, Icarus, 172,402

Osip, D. J., Kern, S. D., \& Elliot, J. L. 2003, Earth Moon \& Planets, 92, 409

Parker, J. W. 2005, The Kuiper Belt Electronic Newsletter, 40, 2

Rickman, H. 1990, in Comet Halley, ed. J. W. Mason (Ellis Horwood, New York), 2, 163

Trujillo, C. A., Jewitt, D. C., \& Luu, J. X. 2000, ApJ, 529, L103

Veillet, C., Parker, J. W., Griffin, I., et al. 2002, Nature, 416, 711

Weissman, P. R. 1995, ARA\&A, 33, 327

Whipple, F. 1986, in Exploration of Halley's Comet ed. B. Battrick, et al., 20th ESLAB Symp., ESA SP-250 II, Noordwijk, 281 